\title{
Implementing airline route map de-cluttering strategies
}

\author{
Paulo Raposo ${ }^{\text {a,*, }}$ Menno-Jan Kraak ${ }^{\mathrm{a},}$ \\ a University of Twente / ITC,p.raposo@utwente.nl,m.j.kraak@utwente.nl \\ * Corresponding author
}

Keywords: airline route maps, visual clutter, visualization strategies

\begin{abstract}
:
Airline route maps show the fights an airline operates between destination airports. Maps like these tend to become cluttered whenever there are more than a few routes. The clutter depends on the amount of flights to display; the routes of flight paths, the balance between shorter (e.g., domestic) and longer (e.g., intercontinental) flights, and the geographic locations of route endpoints. Flights are typically shown as semantically uniform, despite having varied characteristics such as departure or arrival times, frequencies, or passenger volumes. Most maps only indicate the existence of a connection, since the main purpose is to impress customers with the connectivity of the airline in question (i.e., the order or size of the network graph, corresponding to the number of locations served and the number of flight connections, respectively).

This project attempts to catalog the various decluttering design strategies that have been used in the past on printed airline route maps, and to build an online viewer for one airline network in particular that both allows for user selection across the strategies identified in order to explore their relative merits, and querying and visualization of useful attribute data, such as flight volumes and frequences.

What are good strategies to design an airline route map? To answer this question, we examined hundreds of airlines maps from the past. From these maps we identified seven distinct clutter-reduction strategies. Some rely on geometric transformations (i.e., typically warping or scaling, or the use of abstract or schematized space), while others use significant artistic freedom in symbol selection and design. We describe each strategy below.
\end{abstract}

1. Projection Selection. A common strategy is to select a map projection that spreads or "unravels" the network. Figure 1.A shows the JAL network in a polar azimuthal projection, in which regions farther away from the north pole are increasingly spread out, opening up map surface space for nodes, edges, and labels. Uncommon projection choices such as this can help deal with networks containing routes that cross polar regions, or that have dense sets of connections in a region whose area the projection expands. This can make space for nodes, edges, and labels.

2. Topographic Manipulation. Segments of the map such as whole continents can be brought closer together, selectively scaled, or displaced to emptier areas of the map, to open up map surface space in a manner that is convenient for the network being drawn. In Figure 1.B, Egypt has been conveniently moved into the Atlantic Ocean to allow for a more spread-out network of routes to locations in Europe. This strategy is frequently used when there are relatively spatially-disparate but densely-packed clusters of endpoints in the network (e.g., multiple European and multiple North American destinations, but little elsewhere), or when moving one or a few topologically-central endpoints (e.g., an airline hub city) away from the mass of its connecting nodes conveniently fans-out the network edges, such as in the provided Egyptian case.

3. Insets and Blow-Ups. The use of a main map for global connections and insets for smaller, busy areas or domestic networks is also frequently used. Figure 1.C displays Pan Am's global network with insets for Europe and the eastern US. This strategy is particularly useful for networks that are "multiscale," in that their routes tend to cluster across short-haul, local tiers and long-haul, distant tiers.

4. Schematization. Schematic maps, where space is selectively distorted to produce relatively rectilinear or angular shapes, and exemplified by many subway and public transport maps, are used as well. Usually, both the networks and the basemaps are schematized. Geographic distortions are varied, but generally ubiquitous. An example is found in 
Figure 1.D, showing Air Canada's network. Geometric distortions can be selected at the designer's convenience, with choices likely driven by the network in question.

5. Leaving Out Basemaps. Some airline route maps only show connections between destinations, leaving out any contextualizing basemap. The layout of network endpoints is not always consistent; they are sometimes planimetrically precise according to some map projection, and sometimes systematically or arbitrarily distributed across the map surface. The New York Air map in figure 1.E is an example. Leaving out basemaps creates more whitespace on the map, which is either useful for drawing network information more densely, or left bare as an aesthetic choice.

6. Abstract Diagrams. Non-map solutions are sometimes used, with diagrammatic techniques to visualize the network, even though these are frequently similar or evocative of maps. The characteristics of the geography in question, such as scale or directions, tend to be lost. Figure 1.F show the connectivity of Turkish Airlines as a radial diagram; the globe in the center is decorative and suggestive only. For reasons similar to schematization, this strategy can be applied to any route network, though it is interesting to note that it is relatively uncommon, perhaps because it tends to not produce maps.

7. Point Locations Only. A simple and minimalist approach sometimes taken is to plot destinations (nodes) on a map while leaving out connection lines (edges). As with leaving out basemaps, this creates more whitespace. Figure 1.G shows an example from Air France.

To benefit from existing design solutions, as well as incorporate attribute data, this project has been developing an online viewing environment that will allow for route de-cluttering method selection and interactive querying and visualization of attributes associated to each route (e.g., volumes, frequency, aircraft model, etc.) for a specific airline network. A screenshot of our viewer in its current state is given in Figure 2. The work is still in its infancy, and we will present advances made at the conference.

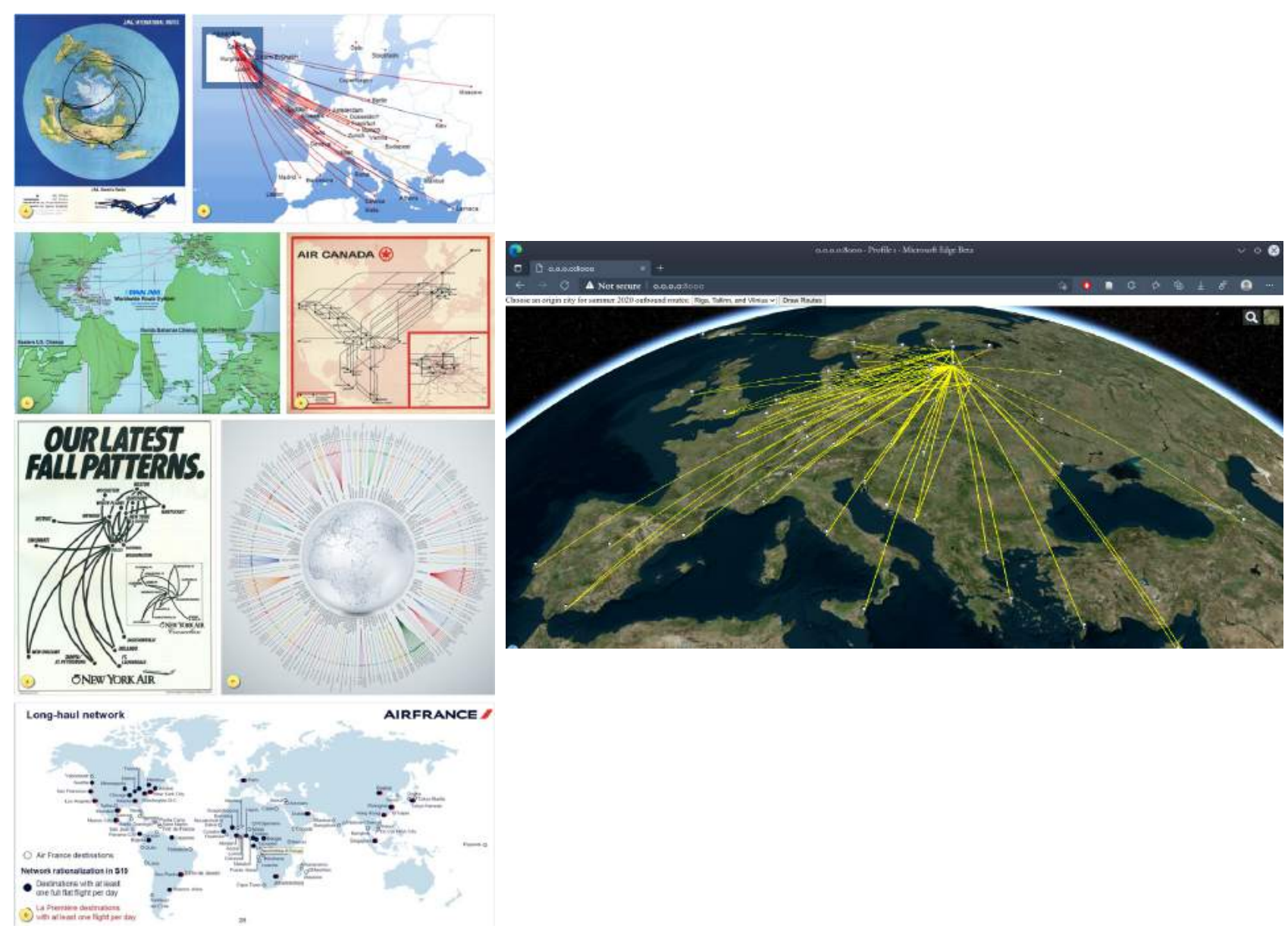

Figure 1 (left). Airline network visualization strategies. Figure 2 (right). Screenshot of our preliminary viewer. 